\title{
Analysis of Different Scalar Probe Compensation Methods for an Array of Near-Field EMI Probes
}

\author{
Tim Claeys, Guy A. E. Vandenbosch Fellow, IEEE and Davy Pissoort Senior Member, IEEE
}

\begin{abstract}
This paper proposes and analyses two possible methods to compensate the raw near-field data obtained with an array of near-field probes. The first method is based on the spatial compensation procedure for a single-probe and is modified towards an array of probes. The second method is to use standard spectral (plane wave) single probe compensation. To use the latter, guidelines on how to design an array and modify the overall measurement procedure such that mutual coupling and edge effects have less influence, are given. The different methods are tested in simulation, and compared with the performance of compensation on a single probe. It is shown, that the standard spectral single probe compensation, in combination with proper array design, will result in an error as low as the same compensation technique used on a single probe. It is also shown that the spatial compensation improves the results in comparison with regular calibration. However the error is not low enough to be used for post-processing due to ill-conditioning. This paper shows the path to much needed, fast and accurate near-field measurements.
\end{abstract}

Index Terms-Near-field scanning, Probe compensation, Probe Array, Convolution

\section{INTRODUCTION}

Over the last two decades, near-field (NF) scanning has proven to be an effective and efficient characterization method within ElectroMagnetic Compatibility (EMC) engineering. Applications include, but are not limited to, location of sources - responsible for excessive radiated emissions - at the Printed Circuit Board (PCB) level [1], [2], visualizing the current flow on a PCB [3], [4], or predicting the far-field emission through near-to-far-field transformation [5], [6]. In many cases, there is a need to know the currents and certainly the far-field with high accuracy, meaning that the near-field data also need to be accurate.

Similar to an antenna, a near-field probe is, in essence, a transducer that transforms the electric and/or magnetic fields, that one wants to measure, into power/voltage/current levels that can be detected with a measurement receiver. Hence, procedures are necessary to turn these received power/voltage/current levels into the correct field values again. Equivalent to the concept of antenna calibration, [7] introduces a probe calibration technique. While this calibration procedure is very easy to understand and implement, it oversimplifies matters if accurate near-field data are needed. Indeed, such a calibration procedure doesn't take into account that a nearfield probe doesn't measure the field in a single point, but in

T. Claeys and D. Pissoort are with the Department of Electrical Engineering, M-Group, KU Leuven Bruges Campus, 8000 Bruges, Belgium.

G. A. E. Vandenbosch is with the Department of Electrical Engineering, Telecommunication and Microwaves, KU Leuven, 3000 Leuven, Belgium.

Manuscript received April 19, 2005; revised August 26, 2015. fact measures a weighted average of the fields in the vicinity of the probe [8]. In [8], this weighted average is expressed as the convolution of the field surrounding the probe with a probe factor. This averaging effect can be reduced by reducing the probe's size. However, this inevitably leads to a lower sensitivity of the probe, resulting in a lower signal-to-noise ratio and a lower dynamic range of the complete near-field scanning set-up [9]. Also note that this effect is not always a problem, for example in MRI scanning [10]-[12] where the probe is at a fixed position, regular antenna calibration [7] is more than enough to get accurate results.

Given the above, it is not surprising that people have proposed methodologies to compensate for the weighted averaging effect. Two different flavours exist, scalar [13] and vector [14] compensation. Both work in the so-called spectral plane wave domain by starting from a Plane Wave Expansion (PWE) [15] of the near-fields. The difference between both is that scalar compensation assumes that the near-field probe only detects one single component of the electromagnetic field (e.g. the vertical electrical field for a vertical dipole), while vector compensation accounts for the fact that a near-field probe is sensitive to all components of the electromagnetic field. Over the years, different additions on these techniques have been presented. e.g. in [16] it is shown how reciprocity in simulations can be used to determine the probe factor. Quite recently, spatial deconvolution has been proposed as an alternative [17]. In that case, the deconvolution is performed directly in the spatial domain instead of going to the spectral (plane wave) domain. While from a computational point of view, spatial deconvolution is more complex, it does have the advantage of being less sensitive to truncation errors when the near-field can only be measured over a small area. In addition, a few compensation techniques based on completely different working principles have been proposed, however, often with less general applicability. To give an example, the weighted average compensation proposed in [18] relies on known current distributions on the probes, which are generally not available for practical near-field EMC measurements.

The weighted averaging effect and the corresponding (lack of) accuracy of the near-field data is not the only practical issue for the wide application of near-field scanning for EMC purposes. Practical EMC near-field measurements also suffer from excessive measurement times. Solutions to this comprise: (i) model-based sequential sampling techniques [19], [20] that select the most optimal measurement points as the measurement progresses instead of simply measuring the near-field data on a full and regular grid, and (ii) application of an array of near-field probes [21]-[23] instead of a single near-field 
probe. Using a smart control system the output of each probe inside the array is multiplexed to the array's main output which can be connected to the measuring device.

Up to now, compensation methods have only been proposed for single near-field probes. This paper wants to bridge the gap with compensation techniques for electrically switched arrays of near-field probes. As such, this paper paves the way for fast and accurate measurements of near-field data, and, hence, accurate post-processing of that data. Due to the finite size of a practical near-field probe array, the procedure developed for single-probe compensation cannot simply be applied. In this paper, two innovative solutions are proposed: (i) a modified version of the spatial deconvolution technique from [17] to arrays and (ii) appropriate design of the array itself such that single probe compensation can be used with acceptable accuracy for near-field measurements with an array of probes. Note that both methods are based on the scalar and not the vector compensation.

Note that the compensation described in this paper is different from the removal of mutual coupling as described in [24]. The compensation method described here removes the unwanted weighted averaging effect of the probes. Also, since it is assumed that the probe measures a single component of the electromagnetic field, cross-polarization as described in [25] is not removed. Though this problem can be tackled in future work using the vector compensation described in [14].

The remainder of this paper is organized as follows. In Section II, the main aspects of scalar compensation for single near-field probes are repeated, which is necessary to understand the following sections. In Section III the first method for the compensation of an array of near-field probes, using compensation in the spatial domain, is presented. The second method, applying the "single probe" spectral compensation on an array, is presented in Section IV. Section V verifies the accuracy and effectiveness of the proposed solutions on several simulated test cases. Finally, Section VI summarizes all results. Note: in this paper the symbol $\mathbb{Z}$ denotes the set of all integers and $*$ denotes the convolution operator.

\section{Scalar Single Probe COMPENSATION}

This paper focusses on the scalar compensation of an array of probes. Therefore the scalar compensation presented in [13] is repeated here in order to understand the new scalar compensation method for an array of probes.

As mentioned in the introduction, in scalar compensation, it is assumed that the probe output only depends on one component of the electromagnetic field. The probe output $p_{v}$ is described as a convolution of the exact (magnetic or electric) field $f_{v}$ and a probe factor $p f_{v}$, with $v$ the chosen component of the electric or magnetic field $(v=x, y$ or $z)$ :

$$
p_{v}(x, y)=f_{v}(x, y) * p f_{v}(x, y)
$$

In the plane wave domain, (1) can be rewritten as a multiplication, with $P_{v}, T_{v}^{f}$ and $P F_{v}$ the spectral plane wave representations of $p_{v}, f_{v}$ and $p f_{v}$, respectively:

$$
P_{v}\left(k_{x}, k_{y}\right)=T_{v}^{f}\left(k_{x}, k_{y}\right) P F_{v}\left(k_{x}, k_{y}\right)
$$

Using this assumption, the error on the probe output due to the convolution effect, can be removed. Compensating for this convolution effect can be done using the following algorithm:

1) Simulate the field distribution of a reference structure $f_{v}^{\text {ref }}(x, y)$

2) Measure the field distribution of the same reference structure $p_{v}^{\mathrm{ref}}(x, y)$

3) Calculate $T_{v}^{f \text {,ref }}\left(k_{x}, k_{y}\right)$ and $P_{v}^{\text {ref }}\left(k_{x}, k_{y}\right)$ via a $2 \mathrm{D}$ Fourier transform

4) Calculate the probe factor $P F_{v}\left(k_{x}, k_{y}\right)=\frac{P_{v}^{\text {ref }}\left(k_{x}, k_{y}\right)}{T_{v}^{f, \text {,ref }}\left(k_{x}, k_{y}\right)}$

5) Measure the field distribution of the Device Under Test (DUT) $p_{v}^{\text {DUT }}(x, y)$ and calculate $P_{v}^{D U T}\left(k_{x}, k_{y}\right)$

6) Use the probe factor $P F_{v}\left(k_{x}, k_{y}\right)$ calculated in step 4) to get the exact DUT field in the plane wave domain $T_{v}^{f, \text { DUT }}\left(k_{x}, k_{y}\right)=\frac{P_{v}^{\text {DUT }}\left(k_{x}, k_{y}\right)}{P F_{v}\left(k_{x}, k_{y}\right)}$

7) After a $2 \mathrm{D}$ inverse Fourier transform, the exact field $f_{v}^{\text {DUT }}(x, y)$ of the DUT is retrieved

Several extra steps can be taken in order to increase the overall accuracy of this technique:

1) Windows: To reduce the truncation error, a spatial window can be added to all spatial values. In [13], [26], [27] it is shown that a Tukey window works best for scalar compensation. This window gradually reduces the values at the edges of the measuring plane to zero and maintains the correct values in the middle (edge values should always be as low as possible since they introduce large errors in the spectrum).

2) Zero-Padding: Since a PWE is used, the assumption of periodicity in the Fourier transform has to be kept in mind. Accounting for this periodicity, zero-padding of the spatial values is needed [13], [28].

3) Filtering: A low-pass filter can be added to the spectral values reducing the unwanted high (spectral) frequency noise [13] removing every value below a deterministically determined signal-to-noise ratio (SNR) value (3).

$$
P F_{v}\left(k_{x}, k_{y}\right)=0 \rightarrow P F_{v}\left(k_{x}, k_{y}\right)<\max \left(P F_{v}\right)-\mathrm{SNR}
$$

Note that when $P F_{v}\left(k_{x}, k_{y}\right)=0, T_{v}^{f \text {,DUT }}\left(k_{x}, k_{y}\right)$ is also set to zero.

In what follows all these extra steps are also implemented using $100 \mathrm{~dB}$ as the SNR for the low-pass filter, unless explicitly mentioned otherwise.

\section{Spatial Probe Array Compensation}

In this section the theoretical definition of the measured probe values of an array in the spatial domain is derived. Using this definition, the measured probe values can be compensated. Let us first start from the situation in which an infinite one dimensional array of identical probes (one row in the $x$ direction) is used to measure a two dimensional near-field plane. The distance between all the probes is the same as the distance between the discrete measurement points defined as $\left(x_{i}, y_{j}\right)$. The output $p_{a}\left(x_{i}, y_{j}\right)$ of a probe located on point $\left(x_{i}, y_{j}\right)$, in a probe array can be described as (4) [29]. Note that the subscript $a$ denotes the output of a probe in an array. 


$$
\begin{aligned}
p_{a}\left(x_{i}, y_{j}\right) & =\cdots S_{x_{i}, x_{i-1}} p\left(x_{i-1}, y_{j}\right) \\
& +S_{x_{i}, x_{i}} p\left(x_{i}, y_{j}\right)+S_{x_{i}, x_{i+1}} p\left(x_{i+1}, y_{j}\right) \cdots \\
& =\sum_{h=-\infty}^{\infty} S_{x_{i}, x_{i}-h} p\left(x_{i-h}, y_{j}\right)
\end{aligned}
$$

In (4) $S_{x_{i}, x_{i}-h}$ denotes the coupling between the probe located at $\left(x_{i}, y_{j}\right)$ and the probe located at $\left(x_{i}-h, y_{j}\right)$. Also, $p\left(x_{i}, y_{j}\right)$ is the probe output as if it was a single probe, located at $\left(x_{i}, y_{j}\right)$. Note that $x_{i}, y_{j}$ is the discrete spatial point indexed with $i, j \in \mathbb{Z}$. In an infinite array $S_{x_{i}, x_{i}-h}=S_{x_{i}-z, x_{i}-h-z} \forall z \in \mathbb{Z}$, so that $S_{x_{i}, x_{i}-h}$ can be written as $S[h]$. The probe output $p_{a}\left(x_{i}, y_{j}\right)$ can then be written as a convolution sum between the mutual coupling and each single probe output:

$$
p_{a}\left(x_{i}, y_{j}\right)=\sum_{h=-\infty}^{\infty} S[h] p\left(x_{i-h}, y_{j}\right)=S * p
$$

Using the discrete form of (1) in (5) results in :

$$
p_{a}=S *(p f * f)=(S * p f) * f=p f_{a} * f
$$

with $p f$ the probe factor of a single probe and $p f_{a}$ the probe factor of the same probe in an infinite array of probes.

As can be seen from (6) and (1) the compensation technique for an infinite array is exactly the same as for single probes. Because infinite arrays are impossible to realize in practice, the array compensation has to work with finite arrays. The edge effects of a finite array will have a large effect on the mutual coupling between the probes in the array. In an infinite array, the coupling between probes is the same regardless of the location in the array. In a finite array there is a difference between the coupling between the probes depending on the location in the array (7).

$$
S_{x_{i}, x_{i}-h} \neq S_{x_{i}-z, x_{i}-h-z} \forall z \in \mathbb{Z}
$$

Let us now turn to the situation in which the probe array is now a real finite one-dimensional array with $G$ probes in the $x$-direction. The distance between the probes is again the same as the distance between the measuring points. In Fig. 1 a part of an array measurement is shown. The black dots represent the points that need to be measured while the red dots show the placement of the array, which in this case is an array of two probes. Four positions of the array placement are shown (a total of 8 positions are needed to fully measure all black dots).

In Pos 1, the two probe outputs can be described by the two equations in (8a) and (8b). Each probe in the probe array has a probe index $g$ indicating which probe is used in the array for the measurement at $\left(x_{i}, y_{j}\right)$. In the measurement point $\left(x_{i}=\right.$ $\left.0, y_{j}=0, g=0\right)$ the probe factor that is used is $p f_{a, v}^{g=0}$ while in the other measurement point $\left(x_{i}=1, y_{j}=0, g=1\right)$ the probe factor of the second probe $p f_{a, v}^{g=1}$ is used .

$$
p_{a, v}(0,0,0)=\int_{-\infty}^{\infty} \int_{-\infty}^{\infty} f_{v}\left(x_{m}, y_{n}\right) p f_{a, v}^{0}\left(x_{m}-0, y_{n}-0\right) d x_{m} d y_{n}
$$

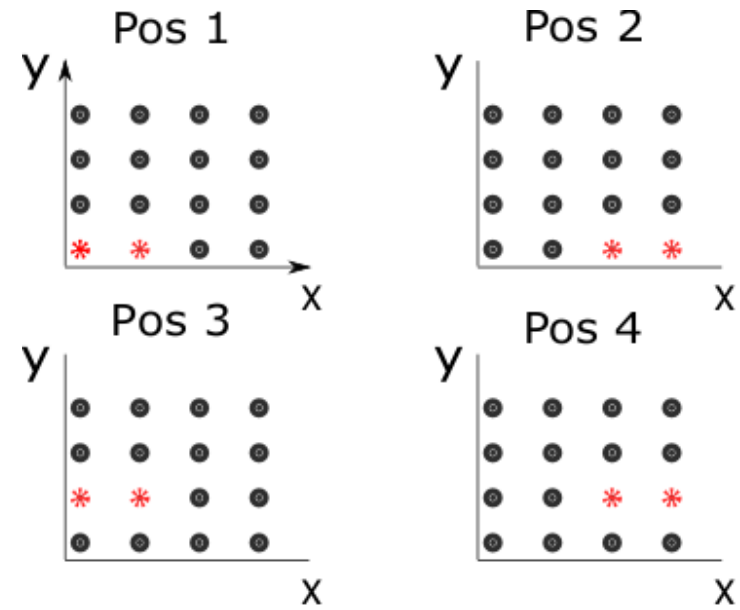

Fig. 1: Array measurement

$$
p_{a, v}(1,0,1)=\int_{-\infty}^{\infty} \int_{-\infty}^{\infty} f_{v}\left(x_{m}, y_{n}\right) p f_{a, v}^{1}\left(x_{m}-1, y_{n}-0\right) d x_{m} d y_{n}
$$

In Pos 2 two equations ((9a) and (9b)) can again be defined that represent the convolution in each measurement point where the array is located.

$$
\begin{aligned}
& p_{a, v}(2,0,0)=\int_{-\infty}^{\infty} \int_{-\infty}^{\infty} f_{v}\left(x_{m}, y_{n}\right) p f_{a, v}^{0}\left(x_{m}-2, y_{n}-0\right) d x_{m} d y_{n} \\
& p_{a, v}(3,0,1)=\int_{-\infty}^{\infty} \int_{-\infty}^{\infty} f_{v}\left(x_{m}, y_{n}\right) p f_{a, v}^{1}\left(x_{m}-3, y_{n}-0\right) d x_{m} d y_{n}
\end{aligned}
$$

Combining all equations of all eight possible positions results in the total measurement definition:

$$
p_{a, v}\left(x_{i}, y_{j}, g\right)=\int_{-\infty}^{\infty} \int_{-\infty}^{\infty} f_{v}\left(x_{m}, y_{n}\right) p f_{a, v}^{g}\left(x_{m}-x_{i}, y_{n}-y_{j}\right) d x_{m} d y_{n}
$$

Since a near-field scanner and array measure in discrete spatial points over a finite domain, the integral in (10) is approximated by a circular discrete convolution, as was shown in [17]. The accuracy of this approximation depends on (i) the number of points $(M, N)$ and (ii) the total size of the spatial area $\left(x_{N}-x_{0}, y_{N}-y\right)$. As a guideline for the number of samples and total spatial area can be found in [30].

$p_{a, v}\left(x_{i}, y_{j}, g\right) \approx \sum_{m=1}^{M} \sum_{n=1}^{N} f_{v}\left(x_{m}, y_{n}\right) p f_{a, v}^{g}\left(x_{m}-x_{i}, y_{n}-y_{j}\right)$

Equation (11) represents the single convolution on a point $\left(x_{i}, y_{j}\right)$ on the measurement plane of the probe factor of the probe $g$ above this point with the exact field surrounding that measurement point. For each measurement point $\left(x_{i}, y_{j}\right)$ in the measurement, as been shown in the equations (8) and (9), an equation can be written down. All these equations can than be combined into a matrix-system:

$$
\left[p_{v}\right]=\left[p f_{a, v}^{g}\right]\left[f_{v}\right]
$$

This matrix-system is the theoretical definition of a one dimensional probe array near-field measurement. It is very 
similar to the matrix system defined in [17]. Solving this system via (13), if each probe factor is known and if the matrix $\left[p f_{v}^{g}\right]$ is invertible, results in .

$$
\left[f_{v}\right]=\left[p f_{a, v}^{g}\right]^{-1}\left[p_{v}\right]
$$

If a two dimensional array is used $p f_{a, v}^{g}$ becomes $p f_{a, v}^{g, h}$ with $h$ the index of the probe that is used in the $y$ direction of the array. In what follows the notation of a 2D array is used for generality. For a one dimensional array $h$ will be a constant. The matrix $\left[p f_{a, v}^{g, h}\right]$ can then be inverted by a direct inversion if the matrix is not singular, or using other techniques, e.g. LQSR, Tikhonov regularisation, etc. Note that the memory in a computer will limit the number of measuring points when these techniques are used. In comparison with single probe compensation the matrix inversion techniques are also much slower.

\section{Spectral Probe Array Compensation}

Another way of approaching the problem of compensating the probe array is, instead of developing a compensation technique for an array of probe factors, to modify the array as such that the single probe compensation technique presented in Section II can be used.

One can not use single probe compensation since $\left(S_{x_{i}, x_{i}-h} \neq S_{x_{i}-1, x_{i}-h-1}\right)$ as described in (7). Instead of searching for a method to solve the system in (13), other methods can be used. A first solution is to make $\left\|S_{x_{i}, x_{i}-h}-S_{x_{i}-1, x_{i}-h-1}\right\|$ small enough so that each probe factor in the array is almost equal. Under the assumption that in real life each probe can almost be made exactly the same, the coupling between the probes in the array is dependent on the frequency, the probes' topology and the distance between the probes. The influence of these parameters and at which point the coupling is low enough to use single probe compensation is examined in Section V-C1.

The second possibility is to omit probes from the array (i.e. probes that are not used become dummy probes). At the sides of the array $S_{x_{i}, x_{i}-h} \neq S_{x_{i}-1, x_{i}-h-1}$, but in the middle of the array the difference between $S_{x_{i}, x_{i}-h}$ and $S_{x_{i}-1, x_{i}-h-1}$ is lower or even negligible. Hence, the probe factors in the middle of the array are more equal to each other than the ones at the sides of the array. Again, the point at which a certain number of omitted probes is enough for using single probe compensation is examined in Section $\mathrm{V}-\mathrm{C} 2$.

\section{Evaluation}

To test both proposed solutions, to compensate the measurement values of an array of probes, a full near-field measurement is modelled in a full-wave solver. In Section V-A the complete simulation setup is discussed, while the actual evaluation of the spatial and spectral method is shown in Sections V-B and V-C, respectively. A comparison between both methods is shown in Section V-D.

\section{A. Simulation setup}

The array of probes is modelled in Numerical Electromagnetics Code (NEC) [31] as simple (wire) dipoles which can be moved over a DUT (for every placement of the probe a new simulation is executed). The array, shown in Figure 3, consists of 10 electric wires loaded with a $50 \mathrm{Ohm}$ load in the middle, which act as a receiving electric dipole, next to each other along the $\mathrm{x}$-axis measuring the electric $\mathrm{z}$-component. The distance between the probes is chosen to be $1 \mathrm{~mm}$ (which is the same as the measuring resolution).
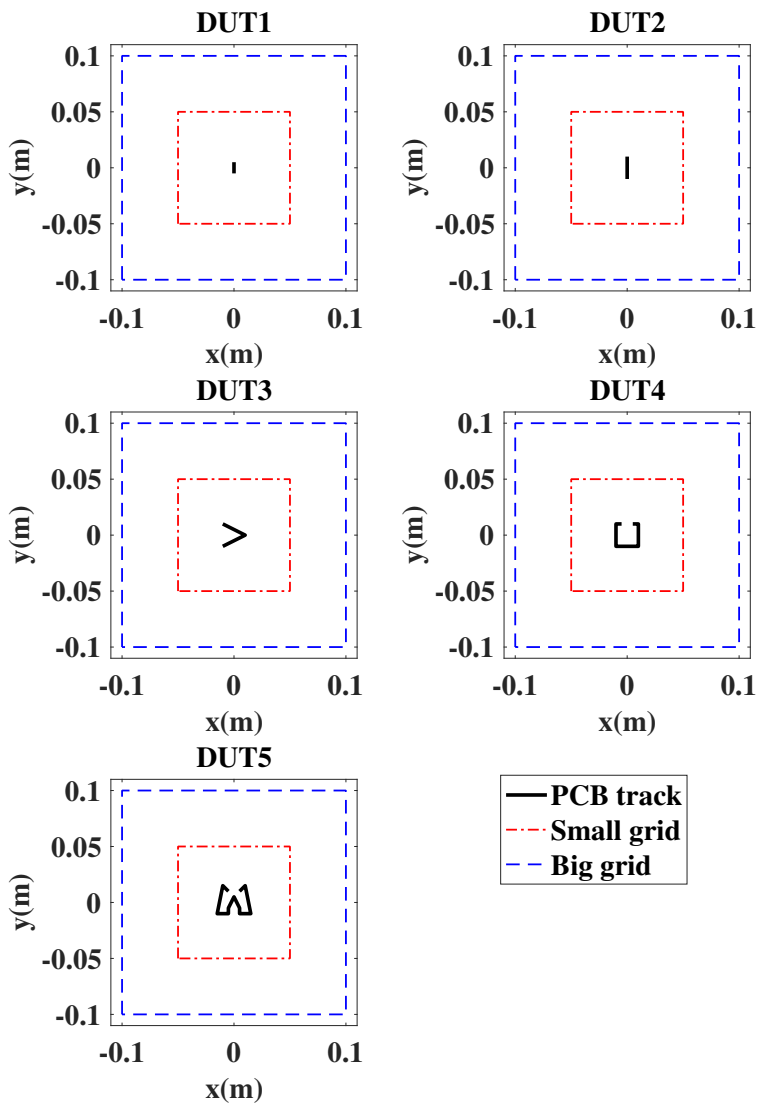

Fig. 2: DUTs modelled in NEC

The DUTs are also made by using simple wires and are placed above an infinite ground plane. Five different DUTs are modelled (shown in Fig. 2). In Fig. 2 the electrical wires are shown in scale with the axes, by the black PCB track. All simulations are done for a frequency of $500 \mathrm{MHz}$ and are all excited with the same $1 \mathrm{~V}-50 \mathrm{Ohm}$ source. All probe factors are calculated on the 'Big grid' shown in Fig. 2 while the compensated DUT values are measured on the 'Small grid'. All simulated measurements are done with a resolution of $1 \mathrm{~mm}$.

In Figure 3 the complete simulation setup (xz-plane) is shown. Each probe is $10 \mathrm{~mm}$ long. The PCB, near field and probe height are $1 \mathrm{~mm}, 8 \mathrm{~mm}$ and $8 \mathrm{~mm}$, respectively. When 
results are compared with a single probe, the single probe has the same size and is positioned at the same height.

For each DUT several simulations are executed:

- At first, the exact NF $f_{v}(x, y)$ for each DUT is calculated.

- In a second simulation, the proposed probe array is used to simulate the measurement $p_{v}(x, y)$ as it would be used in a "real-life" measurement, shown in Fig. 1. To retrieve each $p f_{a, v}^{g, h}(x, y)$, a full simulated measurement per probe in the array is necessary. These full simulated measurements come down to measuring each spatial point in the measurement grid with each and every probe. Note that this has to be performed only once in a real array scanning setup.

Using these simulation values, the two different methods are tested and compared.

\section{B. Spatial Probe Array Compensation}

Several methods exist to invert the matrix in (13). Here, different methods are tested in their performance of inverting $\left[p f_{a, v}^{g, h}\right]$ and using it to de-convolute $p_{a, v}$. The performance of these methods is evaluated using the Mean Squared Error (MSE) (14), visual confirmation and the overall computation time.

$$
\operatorname{MSE}_{z}=\frac{1}{n} \sum_{i=1}^{n}\left(\left|E_{z}^{c o m p}-E_{z}\right|\right)^{2}
$$

In this paper, the following methods are implemented and used in MATLAB [32] to solve (13):

- Least Squares method (LSQR)

- SVD using Tikhonov regularisation [17], [33]

Each PCB shown in Fig. 2 can act as a reference structure (i.e. it can be used to calculate the probe factor of the probe(s)) as well as DUT. In Table I the average error (with DUT1 as a reference structure) of the PCBs other than the reference structure is shown.

All these algorithms are executed in Matlab on a DELL precision 7910 workstation comprising two Intel XEON E5$2620 \mathrm{~V} 4$ processors and $2400 \mathrm{MHz}$ DDR4 RAM. The average calculation time is the average time to finish not only the algorithm but also including the time to build the probe factor matrix $\left[p f_{a, v}^{g, h}\right]$. Note that $\left[p f_{a, v}^{g, h}\right]^{-1}$ only needs to be calculated once per grid size of the DUT.

TABLE I: Comparison solving (13)

\begin{tabular}{c|c|c|c} 
Probe & Method & $\begin{array}{c}\text { Average MSE error } \\
\text { ref = DUT1 }\end{array}$ & $\begin{array}{c}\text { Average } \\
\text { calculation } \\
\text { time (s) }\end{array}$ \\
\hline \hline Array & LSQR & 0.25 & 75.29 \\
\hline Array & Tikhonov & 0.02 & 688.04
\end{tabular}

Applying the spatial method on the simulated measurement results from an array, results in similar error values across the different inversion methods. The calculation time, on the other hand, differs a lot. The LSQR algorithm proves not to be the best solution for inverting the matrix $\left[p f_{a, v}^{g, h}\right]^{-1}$, while inversion with Thikhonov regularisation provides the best results, but at the cost of approximately increasing the calculation time with a factor 10 . Fig. 4 shows a slice $(y=0)$ of the results for different inversion methods. The standard calibration as presented in [7] is added to show the effect of compensation in comparison with regular calibration. Note that the calibration method is applied to every probe in the array separately. Fig. 4 clearly shows that the Thikonov algortihm produces much better results than the LSQR algorithm and the standard calibration.

\section{Spectral Probe Array Compensation}

The second method of compensating a measurement from an array of probes, described in Section IV can only be applied under certain conditions. These conditions are presented in the following subsections.

1) Decreasing the array resolution: The coupling between probes depends on the exact build-up of the array. As described in Section V-A the coupling depends on the frequency, distance between the probes (array resolution) and the topology of the probes. In this evaluation the topology and frequency are kept the same but the distance between the probes is changed. The coupling influence on probe compensation is directly visible in the probe factors of the different probes in the array. The larger the coupling, the larger difference between the probe factors.

In Fig. 5 the deviation between all probe factors $p f_{a, v}^{g, h}(x, y)$ (spatial representation of the probe factor), retrieved from DUT1, are shown. Instead of plotting each probe factor separately, the envelope of all $p f_{a, v}^{g, h}(x, y)$ in the array, for a given array resolution, is plotted. From this figure it is clear that a smaller distance between the probes results in a higher deviation of the probe factors (larger envelope in the plot). When the distance between the probes is larger the deviation of the probe factors lowers and should therefore become more suitable for single probe compensation.

In Fig. 6 the single probe compensation is used on array data. The reference structure is again DUT1 while the DUT is DUT5. It is found that when the resolution of the standard array, as described in V-A, is large, the single probe compensation on these arrays is much better. An increase of distance between the probes from $1 \mathrm{~mm}$ to $5 \mathrm{~mm}$ and $10 \mathrm{~mm}$ already shows satisfying results. The exact error values are compared in Section V-D.

2) Omitting probes at the edge of the array: As mentioned before, the coupling between probes in an array is different at the edges of the array. Given an array of 10 equal probes as described in Section V-A, the effect of the difference in coupling is directly visible in the probe factors of the array. In Fig. 7 all 10 probe factors are shown. Notice the large difference of the probe factors 1,2,3,8,9 and 10 in comparison with probe factors 4,5,6 an 7. Instead of measuring with all 10 probes, measurements are done with 8,6,4 and 2 probes while not using the outer most probes in the array. Note that the probes are not actually removed, but are made passive. Hence, the data from those omitted probes is discarded.

Fig. 8 shows the MSE (14) decreasing when lesser probes are used (not using the probes at the edge of the array). Of 


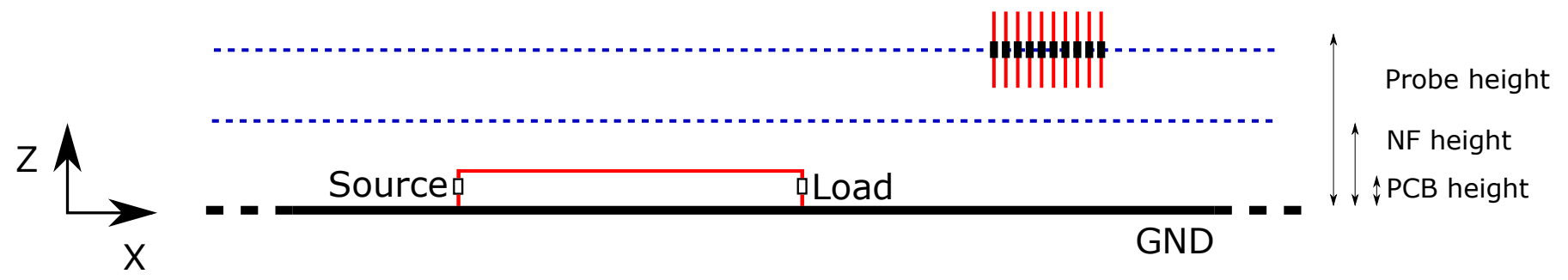

Fig. 3: Simulation representation

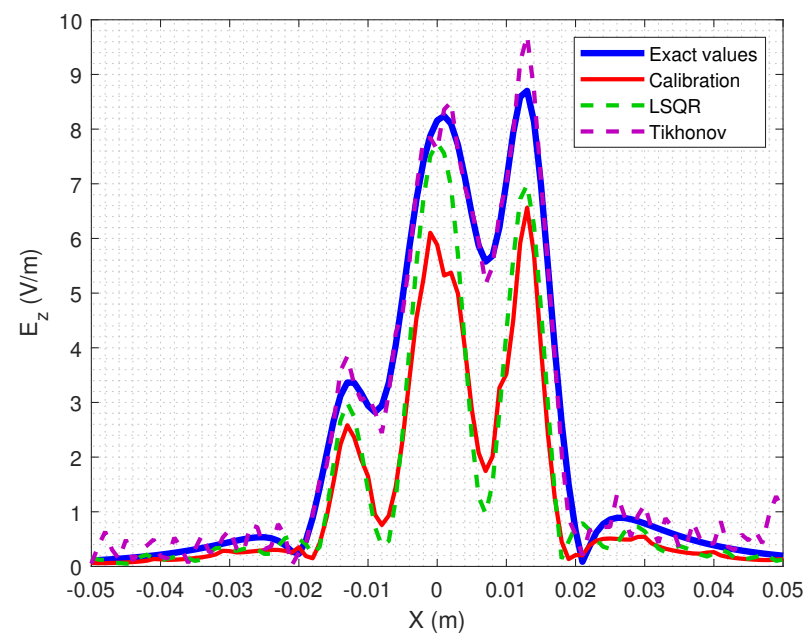

Fig. 4: Comparison compensation results for LSQR and Tikhonov algorithms on an array

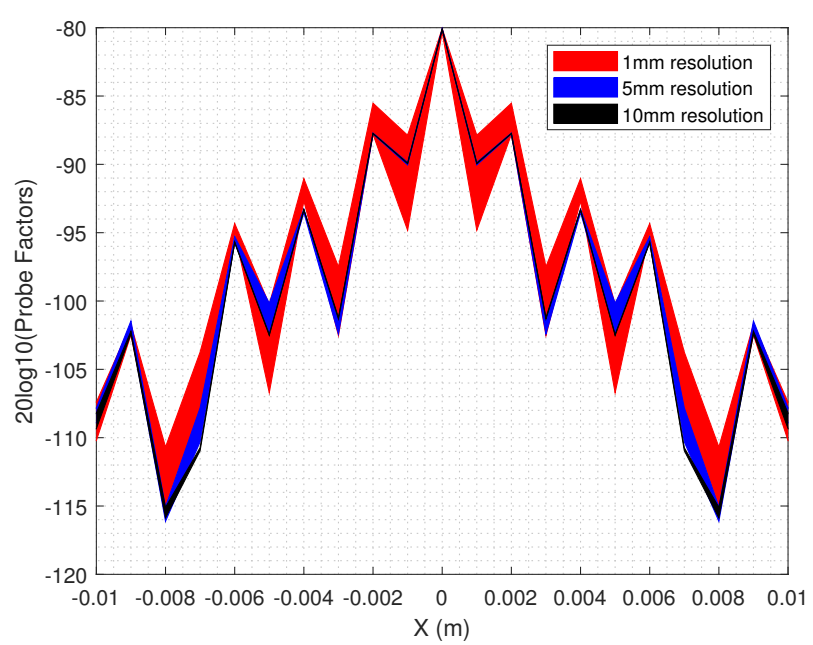

Fig. 5: Probe factor envelopes for different array resolutions

course not using all the probes in the array leads to a higher measuring time in comparison with using all of the probes but this technique does still have a large time advantage in comparison with a single probe. In Fig. 8 the number of omits $(0,1,2,3$ and 4$)$ are the number of probes omitted at one side of the array. The probes at the other side of the array are also omitted. Omits 4, for example, results in only using the two

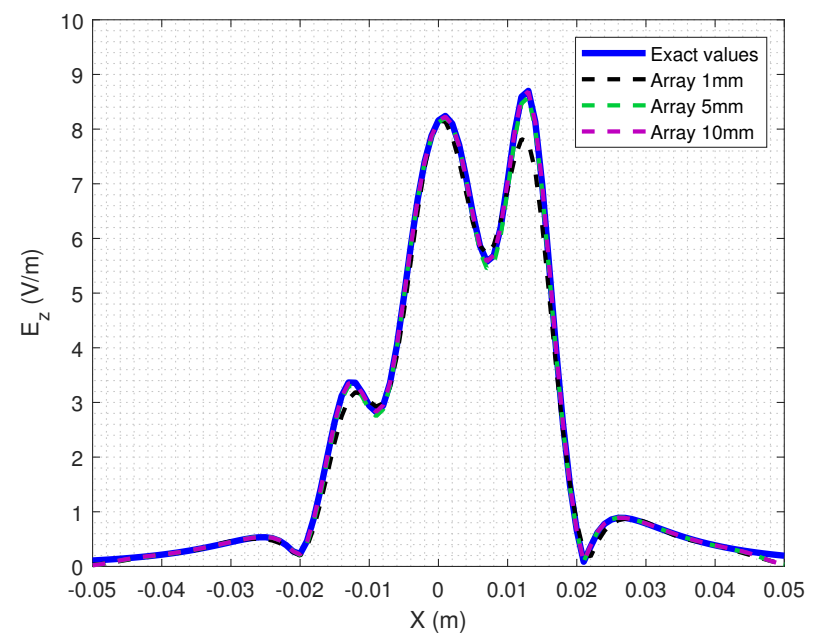

Fig. 6: Array resolution influence on spectral probe array compensation

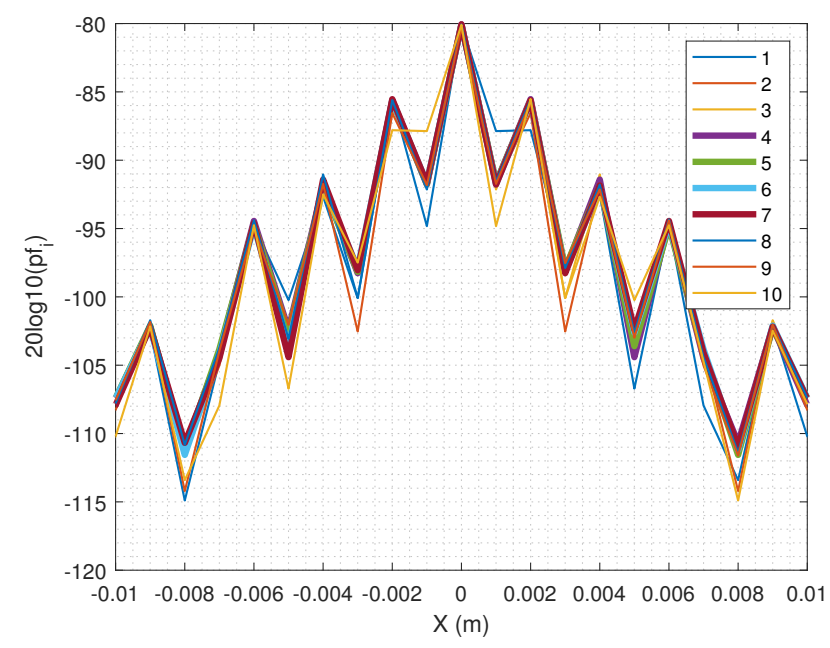

Fig. 7: All probe factors in an array

middle probes in the array.

\section{Comparison of all possible techniques}

In this paper two methods have been presented in order to compensate an array of near-field probes. In Fig. 9 all compensation results from previous subsections are gathered. The application of the two methods on a single probe has 


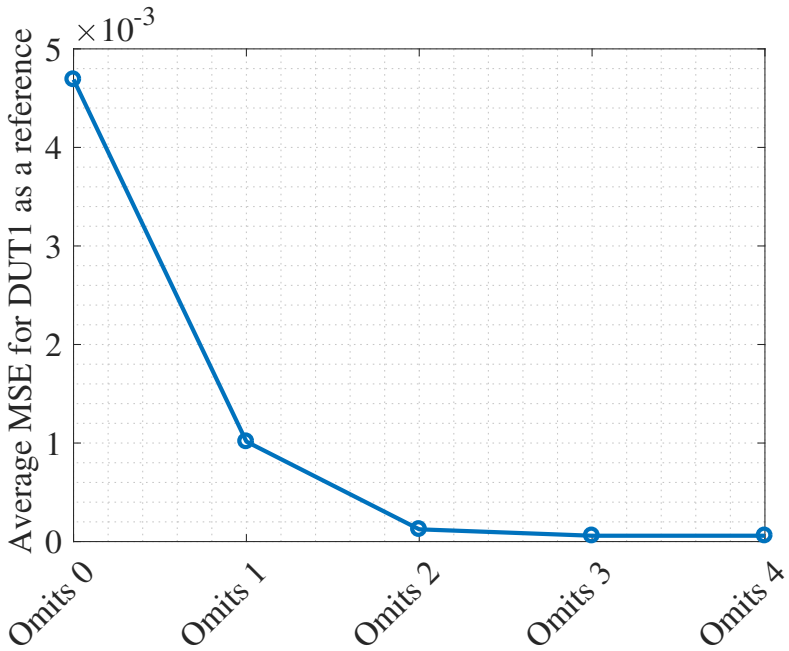

Fig. 8: Probe omit influence on spectral probe array compensation

also been added as a baseline. In this figure, three areas are created that show the subjective opinion of the authors on the compensation results. It is clear that the spectral based method shows the best results, but cannot simply be used for any type of array of probes. The method described in Section III using Tikhonov regularisation or the direct inverse has similar results as the spatial single probe compensation from [17].

It should be noted that the error on the spatial compensation of the single probe is larger in comparison with the spectral compensation method described in Section II, while in [17], spatial compensation shows better results. This is because the spatial compensation technique has an advantage in one specific case. It can compensate measured field values without the need of low level fields at the edges of the measurement [17]. Spectral probe compensation techniques use Fourier to transform the spatial field values to the plane wave domain. High level fields at the edges of the measurement can cause high truncation errors in the compensated results when using a plane wave expansion, as presented in [27].

The error of the spatial methods on the array of probes is of the same order of magnitude as the spatial methods applied to a single probe. The method for spatial array compensation described in Section III is therefore as good as the spatial compensation described in [17] but now applied to an array of near-field probes, enabling a faster measurement with comparable accuracy as a single probe compensated using a spatial deconvolution method. Though, the error is not low enough for good post-processing (note that an error is low enough if it is close to the error of single probe spectral compensation). The remaining error of the spatial method originates from the fact that $\left[p f_{a, v}^{g}\right]$ is ill-conditioned due to noise and errors in the obtained probe factors. Also no proper filtering [34] is applied in the spatial compensation deconvolution technique [17].

The standard spectral based method, which does apply easy to implement filtering, could be used on an array of probes. But again, the error is not low enough. The latter originates from the fact that the convolution theorem assumes a Linear Time Invariant (LTI) system or in the case of nearfield measurements a Linear Place Invariant (LPI) system. In the case of an array measurement, every measurement location can have a separate probe factor. Hence, the measurement can be seen as a Linear Place Variant (LPV) system and the convolution theorem is no longer valid. But, by modifying and carefully designing the array of probes it can come close to a LPI system and one can use single probe compensation on an array of probes. Another reason for using the spectral method is the speed of which the deconvolution is executed in comparison with the spatial method. Most noticeably is the influence of the distance between the probes in the array on the probe factor. If the probe distance goes from $1 \mathrm{~mm}$ to $10 \mathrm{~mm}$, the error drops by a factor $10^{2}$.

\section{CONCLUSION}

In this paper, two methods of scalar probe compensation, involving both a single probe and an array of probes, have been executed and compared. It can be concluded that the array expansion method of the spatial compensation works as good as the spatial compensation for a single probe. The authors of this paper emphasize the need for a correct design of the array of probes. The designer of the array should choose between not using a number of probes at the side of the array or should decrease the array resolution. The time benefit of the array cannot be disregarded. Using the suggestions made in the paper one can now measure the near-field of a PCB very fast and get good accuracy of magnitude after the probe compensation. The authors would also like to mention that in very large arrays reflections could interfere with existing nearfield and cause new accuracy problems. This effect, which is not present with a small array as used in this work, is part of future work. Other future work includes expanding this method and design guidelines for vector compensation, reporting on the results in a wider frequency band, etc.

\section{REFERENCES}

[1] D. Baudry, C. Arcambal, A. Louis, B. Mazari, and P. Eudeline, "Applications of the near-field techniques in emc investigations," Electromagnetic Compatibility, IEEE Transactions on, vol. 49, no. 3, pp. 485-493, August 2007.

[2] K. Haelvoet, S. Criel, F. Dobbelaere, L. Martens, P. De Langhe, and R. De Smedt, "Near-field scanner for the accurate characterization of electromagnetic fields in the close vicinity of electronic devices and systems," in Instrumentation and Measurement Technology Conference, 1996. IMTC-96. Conference Proceedings. Quality Measurements: The Indispensable Bridge between Theory and Reality., IEEE, vol. 2, 1996, pp. 1119-1123 vol.2.

[3] Y. Alvarez, M. Rodriguez, F. Las-Heras, and M. Hernando, "On the use of the source reconstruction method for estimating radiated emi in electronic circuits," Instrumentation and Measurement, IEEE Transactions on, vol. 59, no. 12, pp. 3174-3183, December 2010.

[4] D. Rinas, S. Niedzwiedz, J. Jia, and S. Frei, "Optimization methods for equivalent source identification and electromagnetic model creation based on near-field measurements," in EMC Europe 2011 York, September 2011, pp. 298-303.

[5] V. Volski, S. Yan, G. A. E. Vandenbosch, T. Claeys, and D. Pissoort, "Auxiliary dipoles to compensate for the finite size of the planar scanning area in near-to-far-field transformations," Electromagnetic Compatibility, IEEE Transactions on, vol. 57, no. 6, pp. 1517-1528, December 2015.

[6] J.-S. Lee, T.-L. Song, J.-K. Du, and J.-G. Yook, "Near-field to far-field transformation based on stratton-chu fomula for emc measurements," in Antennas and Propagation Society International Symposium (APSURSI), 2013 IEEE, July 2013, pp. 606-607. 


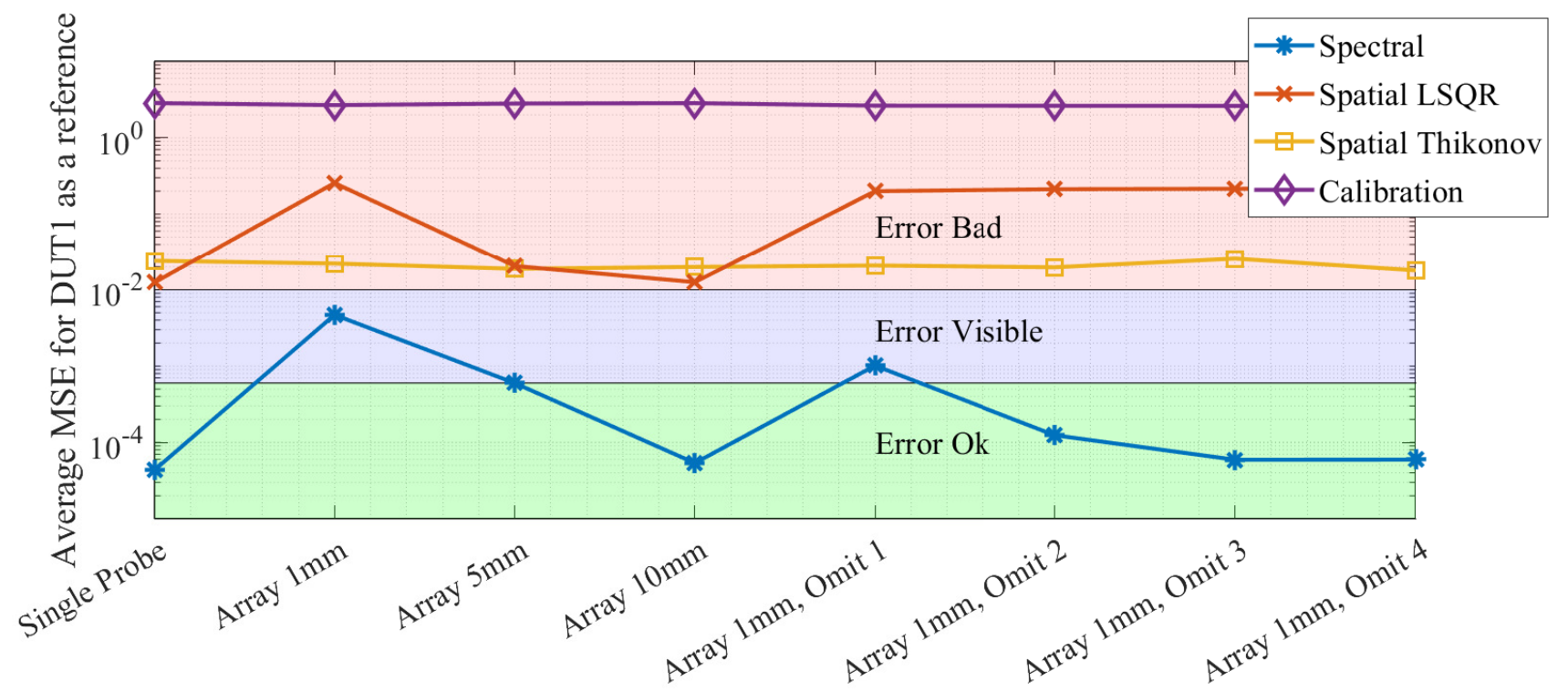

Fig. 9: Comparison scalar compensation on array

[7] IEC/TS, "61967-3 Integrated Circuits - Measurement of electromagnetic emissions - part 3: Measurement of radiated emissions - Surface scan method," 2013.

[8] D. Paris, J. Leach, W., and E. Joy, "Basic theory of probe-compensated near-field measurements," Antennas and Propagation, IEEE Transactions on, vol. 26, no. 3, pp. 373-379, May 1978.

[9] N. Sivaraman, F. Ndagljlmana, M. Kadi, and Z. Riah, "Broad band pcb probes for near field measurements," in 2017 International Symposium on Electromagnetic Compatibility - EMC EUROPE, September 2017, pp. 1-5.

[10] A. Attaran, W. B. Handler, and B. A. Chronik, "2 mm radius loop antenna and linear active balun for near field measurement of magnetic field in mri-conditional testing of medical devices," IEEE Transactions on Electromagnetic Compatibility, vol. 62, no. 1, pp. 186-193, 2020.

[11] A. Attaran, W. B. Handler, K. Wawrzyn, and B. A. Chronik, "Electric field probe for time-domain monitoring of radio frequency exposure during development and evaluation of mri-conditional medical devices at 3 t," IEEE Transactions on Antennas and Propagation, vol. 67, no. 3 , pp. 1854-1861, 2019.

[12] A. Attaran, W. B. Handler, K. Wawrzyn, R. S. Menon, and B. A Chronik, "Reliable rf b/e-field probes for time-domain monitoring of em exposure during medical device testing," IEEE Transactions on Antennas and Propagation, vol. 65, no. 9, pp. 4815-4823, 2017.

[13] A. Tankielun, H. Garbe, and J. Werner, "Calibration of electric probes for post-processing of near-field scanning data," in Electromagnetic Compatibility, 2006. EMC 2006. 2006 IEEE International Symposium on, vol. 1, August 2006, pp. 119-124.

[14] J. Shi, M. Cracraft, K. Slattery, M. Yamaguchi, and R. DuBroff, "Calibration and compensation of near-field scan measurements," Electromagnetic Compatibility, IEEE Transactions on, vol. 47, no. 3, pp. 642-650, August 2005.

[15] P. C. Clemmow, The Plane Wave Spectrum Representation of Electromagnetic Fields:(Reissue 1996 with Additions). Wiley-IEEE Press, 1997, pp. 1-1. [Online]. Available: http://ieeexplore.ieee.org/xpl/articleDetails.jsp?arnumber $=5733172$

[16] H. Weng, D. Beetner, and R. DuBroff, "Frequency-domain probe characterization and compensation using reciprocity," Electromagnetic Compatibility, IEEE Transactions on, vol. 53, no. 1, pp. 2-10, February 2011.

[17] M. Schmidt and M. Albach, "The advantages of spatial domain probe compensation technique in emc near-field measurements," in Electromagnetic Compatibility, Tokyo (EMC'14/Tokyo), 2014 International Symposium on, May 2014, pp. 509-512.

[18] P. Petre and T. K. Sarkar, "Planar near-field to far-field transformation using an array of dipole probes," IEEE Transactions on Antennas and Propagation, vol. 42, no. 4, pp. 534-537, April 1994.

[19] D. Deschrijver, F. Vanhee, D. Pissoort, and T. Dhaene, "Automated nearfield scanning algorithm for the EMC analysis of electronic devices,"
IEEE Transactions on Electromagnetic Compatibility, vol. 54, no. June 2012, pp. 502-510, 2012.

[20] T. Claeys, D. Pissoort, D. Deschrijver, I. Couckuyt, and T. Dhaene, "Sequential sampling algorithm for simultaneous near-field scanning of amplitude and phase," 2014 International Symposium on Electromagnetic Compatibility, pp. 79-84, 2014.

[21] J. C. Bolomey, B. J. Cown, G. Fine, L. Jofre, M. Mostafavi, D. Picard, J. P. Estrada, P. G. Friederich, and F. L. Cain, "Rapid near-field antenna testing via arrays of modulated scattering probes," IEEE Transactions on Antennas and Propagation, vol. 36, no. 6, pp. 804-814, June 1988.

[22] Q. Chen, K. Sawaya, T. Habu, and R. Hasumi, "Simultaneous electromagnetic measurement using a parallel modulated probe array," IEEE Transactions on Electromagnetic Compatibility, vol. 49, no. 2, pp. 263269, May 2007.

[23] B. Archambeault, "Predicting emi emission levels using emscan," in Electromagnetic Compatibility, 1993. Symposium Record., 1993 IEEE International Symposium on, August 1993, pp. 48-50.

[24] J. T. Case, M. T. Ghasr, and R. Zoughi, "Correcting mutual coupling and poor isolation for real-time 2-d microwave imaging systems," IEEE Transactions on Instrumentation and Measurement, vol. 63, no. 5, pp. 1310-1319, May 2014.

[25] "IEEE recommended practice for near-field antenna measurements," IEEE Std 1720-2012, pp. 1-102, December 2012.

[26] T. Claeys, G. A. E. Vandenbosch, and D. Pissoort, "A study of the effects of truncation errors on the compensation of emi near-field probes," in 2016 International Symposium on Electromagnetic Compatibility - EMC EUROPE, September 2016, pp. 23-28.

[27] T. Claeys, V. Volski, G. A. E. Vandenbosch, and D. Pissoort, "Near-field edge extrapolation using auxiliary dipoles to improve probe compensation," IEEE Transactions on Electromagnetic Compatibility, vol. 59, no. 2, pp. 576-583, April 2017.

[28] R. C. Gonzalez and R. E. Woods, Digital Image Processing, 2nd ed. Prentice Hall, 2001.

[29] H. T. Hui, "A new definition of mutual impedance for application in dipole receiving antenna arrays," IEEE Antennas and Wireless Propagation Letters, vol. 3, no. 1, pp. 364-367, December 2004.

[30] E. Joy and D. Paris, "Spatial sampling and filtering in near-field measurements," Antennas and Propagation, IEEE Transactions on, vol. 20, no. 3, pp. 253-261, May 1972.

[31] J. Burke and A. Poggio. (2002) NEC2. [Online]. Available: www.nec2.org

[32] "Matlab 9, the mathworks inc." www.mathworks.com/products/matlab, 2017.

[33] P. C. Hansen, "Regularization tools: A matlab package for analysis and solution of discrete ill-posed problems," Numerical Algorithms, vol. 6, pp. 1-35, March 1994.

[34] S. M. Riad, "The deconvolution problem: An overview," Proceedings of the IEEE, vol. 74, no. 1, pp. 82-85, January 1986. 


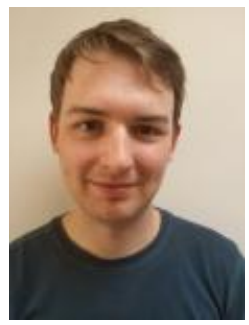

Tim Claeys was born in 1990. He received the M.S. industrial engineering sciences, option electronics, from the University College Katholieke hogeschool Sint-Lieven Gent (KAHO Sint-Lieven), Ghent, Belgium, in 2013. He received the Ph.D. Degree from KU Leuven, Leuven, Belgium, in 2018. Since 2018, he is a Post-doctoral researcher at the M-Group research group (lab FMEC), KU Leuven Bruges Campus, which is a laboratory focusing on global reliability of electronic systems and Electromagnetic Compatibility. His research interests are Near-field scanning, the development of characterization methods for shielding materials and gaskets, EMI resilience of wireless protocols and global reliability of electronic systems.

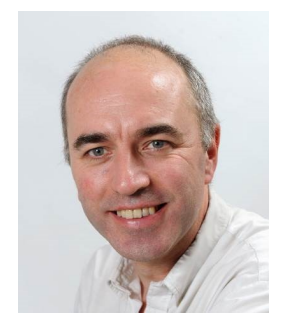

Guy A. E. Vandenbosch received the M.S. and Ph.D. degrees in Electrical Engineering from the Katholieke Universiteit Leuven, Leuven, Belgium, in 1985 and 1991, respectively. Since 1993, he has been a Lecturer, and since 2005, a Full Professor at the same university. His research interests are in the area of electromagnetic theory, computational electromagnetics, planar antennas and circuits, nanoelectromagnetics, EM radiation, EMC, and bioelectromagnetics. His work has been published in ca. 340 papers in international journals and has led to ca. 390 papers at international conferences. Guy Vandenbosch has been a member of the "Management Committees" of the consecutive European COST actions on antennas since 1993. Within the ACE Network of Excellence of the EU (2004-2007), he was a member of the Executive Board and coordinated the activity on the creation of a European antenna software platform. After ACE, from 2007 - 2018, he chaired the EuRAAP Working Group on Software. In the period 1999-2004, he was vice-chairman, in the period 2005-2009 secretary, and in the period 2010-2017 chairman of the IEEE Benelux Chapter on Antennas en Propagation. In the period 2002-2004 he was secretary of the IEEE Benelux Chapter on EMC. In the period 2012-2014, he was secretary of the Belgian National Committee for Radio-electricity (URSI), where he is also in charge of commission E. Guy Vandenbosch is a fellow of the IEEE. From September to December 2014, he was a visiting professor at Tsinghua University, Beijing, China. Since 2017 he is a member of the IEEE Electromagnetics Award Committee.

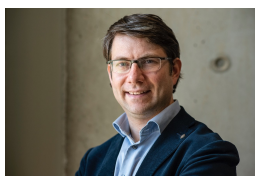

Davy Pissoort (M09SM13) was born in 1978. He received the M.S. and Ph.D. degrees in electrical engineering from Ghent University, Ghent, Belgium, in 2001 and 2005, respectively. From October 2005 to October 2006, he was a Postdoctoral Researcher at Ghent University. From November 2006 to July 2009, he was a Research Engineer in the EesofEDA Department, Agilent Technolgies, Belgium. From August 2009 to September 2016, he was an Assistant Professor and since October 2016 an Associate Professor, both at KU Leuven, Belgium. His current research interests include the development of fast and efficient electromagnetic modeling methods for EMC, SI, and PI, the development of characterization methods for shielding materials and gaskets, EMI Resiliene, dependability of autonomous systems as well as the analysis and testing of the mechanical and thermal reliability of electronic modules. Dr. Pissoort is a Senior Member of the IEEE EMC Society, IEEE EMC Society Board Member and member of the International Steering Committee of EMC Europe. 